\title{
Marketing digital: una herramienta para potenciar y promocionar las Mypimes en el ámbito internacional
}

\section{Digital Marketing: a tool to promote and promote Mypimes in the international arena}

Nancy María Armijos-Delgado

Universidad Internacional del Ecuador, Ecuador

Autor para correspondencia: naarmijosde@uide.edu.ec

Fecha de recepción: 02 de agosto de 2018 - Fecha de aceptación: 01 de diciembre de 2018

Resumen: Esta investigación se efectuó en la ciudad de Loja, en marzo de 2018, dirigido al grupo de empresarios de Mipymes como potencial exportador. La Universidad Internacional del Ecuador extensión Loja estableció un convenio para capacitar a las Mipymes adscritas a ProEcuador. Participaron cinco empresas de las provincias de Loja, El Oro y Zamora Chinchipe; los temas de la capacitación se enfocaron a: estructura de las empresas en el ámbito legal, conocimiento de los productos y servicios, importancia de la marca, logo, slogan, mecanismos para la difusión a través de web y manejo de herramientas de marketing digital, en las que las Mipymes tienen la mayor debilidad. El objetivo fue potenciar y promocionar sus negocios a través de levantamiento de información de las Mipymes y el diagnóstico del estado actual sobre el marketing digital y manejo de tecnologías, herramientas necesarias para competir en mercados internacionales; se destaca la importancia de vincular la academia al servicio empresarial, trabajando en talleres, al brindar una formación de asesoría y capacitación especializada a cada uno de los participantes, orientada a vigorizar la presentación y marca de sus productos hacia el mercado internacional.

Palabras clave: mipymes; herramientas de marketing; marketing digital; comunicación; internet

Abstract: The present research work was developed in the city of Loja, in the period of March 2018, addressed to the group of entrepreneurs of MSMEs as potential exporters. Universidad International del Ecuador, Loja extension, established an agreement to train MSMEs assigned to PROECUADOR. Involving five companies from the provinces of Loja, El Oro and Zamora Chinchipe; the training topics were focused on: structure of companies in the legal field, knowledge of products and services, importance of the brand, logo, slogan, mechanisms for dissemination through the web and management of digital marketing tools, in which MSMEs have the greatest weaknesses. The objective was the gathering of information from MSMEs and the diagnosis of the current state of digital marketing and technology management, to promote and promote their businesses; tools necessary to compete in international markets; the importance of linking the academy to the business service is highlighted, working in workshops by providing specialized training and advice to each of the participants, aimed at strengthening the presentation and the brand of their products, enabling to promote them internationally.

Key words: MSNEs; marketing tools; digital marketing; communication; internet 


\section{Introducción}

La Universidad Internacional del Ecuador, en atención a la disposición del Art. 87 de la Ley de Educación Superior, publicada en el Registro Oficial No. 298 de 12 de octubre de 2010, creó la Comisión de Vinculación con la Sociedad, que se encarga de establecer nexos con instituciones públicas y privadas para el ofrecimiento de planes y programas de capacitación, como el establecido en 2017 con ProEcuador.

Por su parte, ProEcuador es el Instituto de Promoción de Exportaciones e Inversiones Extranjeras, entidad adscrita al Ministerio de Comercio Exterior, que es la delegada de ejecutar las políticas y normas de promoción de exportaciones e inversiones del país, con el fin de promover la oferta de productos tradicionales y no tradicionales, propiciando la inserción estratégica en el comercio internacional.

Los objetivos específicos consistieron en:

- Potenciar y promocionar los negocios mediante las herramientas del marketing digital.

- Diagnosticar sobre la utilización del marketing digital y manejo de tecnologías.

- Capacitar en temas de herramientas digitales para ingresar a competir en el mercado digital clave de alta competitividad y éxito empresarial.

- Capacitar para el diseño e implementación de herramientas digitales de comercialización.

\section{Fundamentación teórica}

Las empresas necesitan de innovación e integración para ingresar en los mercados, con visión estratégica y herramientas que apoyen al desarrollo empresarial; las nuevas tecnologías que aportan al comercio electrónico, oportunidades con dinamismo y flexibilidad, son formas de establecer vías de comunicación con estrategias de uso de redes sociales y comunicación para conocimiento con los clientes.

Además, tomar decisiones estratégicas de éxito y detectar oportunidades, en redes digitales y herramientas de marketing, información necesaria para llegar al mercado meta, de acuerdo a los objetivos del negocio, análisis situacionales digitales, con objetivos claros de marketing, estrategias y tácticas que se van a utilizar para ser competitivos en el mercado nacional e internacional.

Los Planes de Marketing sirven en los procesos de gestión para obtener lo que se propone, necesita guiarse en los planes operativos de la empresa, contribuyendo desde el análisis, planificación, gestión y el control de los procesos de marketing para ofrecer productos con valor. La finalidad de la empresa es vender y el marketing es la vía correcta para conseguirlo, aumentando la productividad con rapidez, seguridad e integración de técnicas y conocimientos de las herramientas de marketing.

En la segunda parte se analizaron los planes avanzados de marketing y comunicación; estrategia digital y planificación de medios tradicionales y digitales; relevancia, comunicación, conveniencia y compromiso a través de la navegabilidad en internet; $y$, conocimientos básicos en 
marketing y estar dispuestos a escuchar sugerencias e invertir en la aplicación de estrategias de marketing digital.

El Plan de Marketing es una herramienta de planificación en el ámbito digital, útil, valiosa y práctica para las Pymes, implica que cada decisión adoptada sea coherente entre sí con el análisis y el diagnóstico realizado, desde la orientación al mercado, a clientes actuales, potenciales, competencia, segmentación y estrategias a utilizar, asegurando la toma de decisiones comerciales, plasmando acciones hacia el rumbo de la actividad comercial, permitiendo fijar objetivos y metas comerciales concretas, asegurando a la empresa una expansión rentable.

Es importante tener en cuenta que estamos en la era digital y el marketing se integra a lo digital para dar respuesta a sus clientes, usuarios y consumidores, para propiciar una mayor interactividad entre los clientes y las empresas, productos y servicios, con nuevas posibilidades de compra, venta; fortaleciendo la relación venta confianza.

En la planificación estratégica de las empresas con el Plan de Marketing digital, la comunicación y posicionamiento es veloz, para conocer por qué los consumidores deciden las compras de productos o servicios, se informan de precios, calidad, diseños, por ello es necesario realizar la inversión en publicidad, específicamente la publicidad digital, como redes sociales, el internet de las cosas, la nube, un mundo más conectado, la economía colaborativa, la inteligencia artificial, productos y servicios con toque humano, realidad aumentada, soluciones diversas de movilidad, impresoras 3D, asistentes virtuales inteligentes, drones. Facilitar la adaptación de las empresas al cambio es una responsabilidad para el marketing.

Para Kotler y Keller (2012), un Plan de Marketing es un documento que recoge los objetivos y estrategias, los planes de acción relativos a los elementos del marketing mix, que facilitarán el cumplimiento de las estrategias.

En la actualidad, para elaborar e implantar un Plan de Marketing hay que considerar que la internet, a través de online, con su gran crecimiento y características específicas ha creado nuevos planteamientos en las estrategias de Marketing (Esteban y Lorenzo, 2013, p. 24).

Buscar una idea de negocio (plan de empresa o negocio) es lo primero que se debe considerar, ya que si no se aplica la empresa puede fracasar antes de empezar, por culpa de una idea mal entendida o poco desarrollada (Zorita y Huarte, 2013, p. 11).

Sainz (2017, p. 47) afirma: "El Marketing digital actual es un proceso responsable orientado a identificar, anticipar y satisfacer las necesidades del cliente, con la finalidad de fidelizarle, de forma que la empresa pueda obtener sus objetivos estratégicos".

El Plan de marketing debe ser un documento de trabajo escrito, ordenado y definido, anual y periódico, "que combine con precisión los elementos del marketing mix y que permita saber cuándo y cómo se han alcanzado los objetivos prefijados en el plan estratégico, estableciendo responsabilidades y poniendo a disposición de la empresa controles flexibles y adaptables a los cambios del mercado" (Fernández, 2000, p. 23-24). 
El plan de marketing es la herramienta de la dirección para plasmar el pensamiento estratégico. Sainz (2017, p. 93) afirma que: "La planificación consiste en el proceso de definición (hoy) de lo que queremos ser en el futuro, apoyado en la correspondiente reflexión y pensamiento estratégico". "El Plan de Marketing constituye un seguro contra el riesgo comercial inherente a la mayor parte de las decisiones comerciales y de marketing" (Sainz, 2015, p. 81). El mismo autor sostiene que "Marketing es un proceso orientado a identificar, anticipar y satisfacer las necesidades del cliente, con la finalidad de fidelizarle, de forma que la Pyme pueda obtener sus objetivos estratégicos" (Sainz, 2016, p. 44).

\section{Metodología}

La metodología comprendió dos fases, que se detallan en las tablas siguientes.

Tabla 1. Nombre de la empresa, localización y productos ofertados

\begin{tabular}{|c|c|c|c|c|}
\hline \multirow[t]{2}{*}{ No. } & \multirow[t]{2}{*}{ Razón social } & \multicolumn{2}{|c|}{ Ubicación } & \multirow[t]{2}{*}{ Productos } \\
\hline & & $\begin{array}{l}\text { Provincia-cantón- } \\
\text { parroquia }\end{array}$ & Dirección & \\
\hline 1 & $\begin{array}{l}\text { Alimentos procesados } \\
\text { y marinados del campo }\end{array}$ & $\begin{array}{l}\text { Loja-Gonzanamá- } \\
\text { Gonzanamá }\end{array}$ & Hacienda El Toldo & $\begin{array}{l}\text { Cuyes, gallinas, } \\
\text { patos marinados }\end{array}$ \\
\hline 2 & $\begin{array}{l}\text { Sombreros de paja } \\
\text { toquilla }\end{array}$ & Loja-Loja-Loja & Calle Indianápolis & $\begin{array}{l}\text { Sombreros de paja } \\
\text { toquilla, artesanías }\end{array}$ \\
\hline 3 & APEOSAE & $\begin{array}{l}\text { Zamora Chinchipe- } \\
\text { Sumbi-Panguintza }\end{array}$ & Panguintza & $\begin{array}{l}\text { Café tostado, cacao } \\
\text { en grano, chifles de } \\
\text { plátano }\end{array}$ \\
\hline 4 & Happy Fruits & $\begin{array}{l}\text { El Oro-Arenillas- } \\
\text { Arenillas }\end{array}$ & $\begin{array}{l}\text { Entrada al barrio } \\
\text { Santa Marianita, vía } \\
\text { Panamericana }\end{array}$ & $\begin{array}{l}\text { Fruta deshidratada: } \\
\text { mango y pitahaya }\end{array}$ \\
\hline 5 & Vinos y Licores del Sur & $\begin{array}{r}\text { Loja-Loja- } \\
\text { Vilcabamba }\end{array}$ & $\begin{array}{l}\text { A media cuadra del } \\
\text { parque central }\end{array}$ & Vinos y licores \\
\hline
\end{tabular}

Fuente: Informe de capacitación marzo 2018

\section{Primera Fase:}

La identificación de las empresas se obtuvo en esta fase, mediante la aplicación de un instrumento diseñado de una ficha de información de las empresas, para determinar la asesoría y capacitación sobre las herramientas de marketing digital y la visita a cada una de las empresas para la verificación de la información y el registro de asistencia, durante el desarrollo de los eventos de capacitación.

En el Convenio se enunció que los beneficiarios serían de 10 a 15 Mipymes adscritas a ProEcuador, sin embargo, concurrieron solamente tres empresas de la provincia de Loja, una de Zamora Chinchipe y una de El Oro, Tabla 1. 


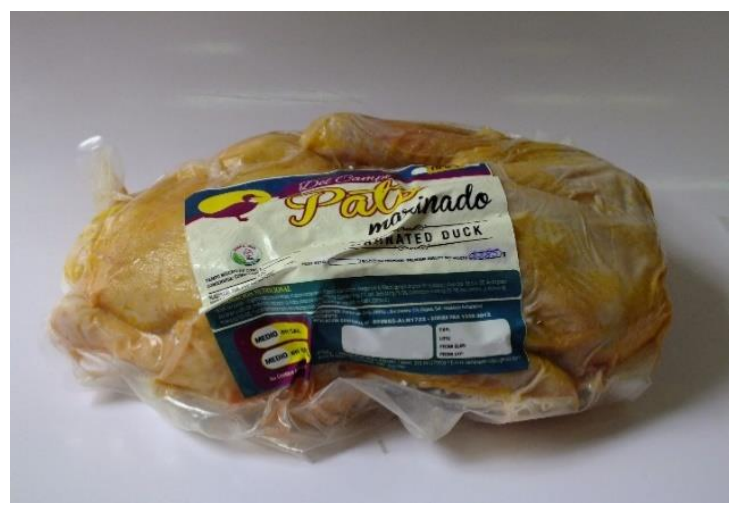

Figura 1. Pato marinado producido por “Alimentos Procesados" de Gonzanamá

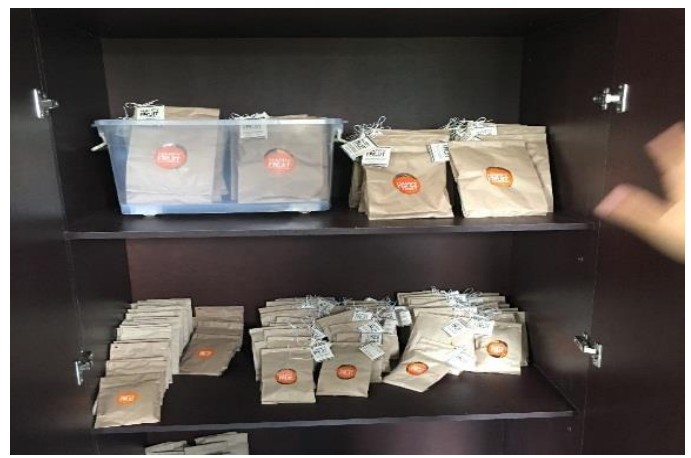

Figura 3. Fundas de snack fabricadas por Happy Fruit de Arenillas, El Oro

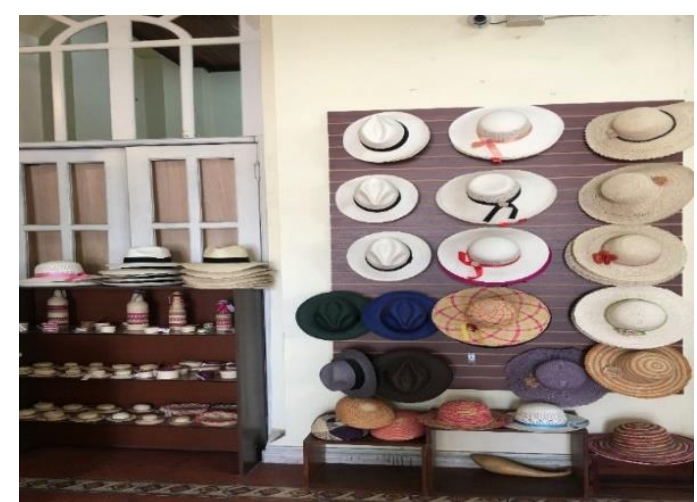

Figura 2. Sombreros artesanales, elaborados por "Sombreros de Paja Toquilla" de Loja

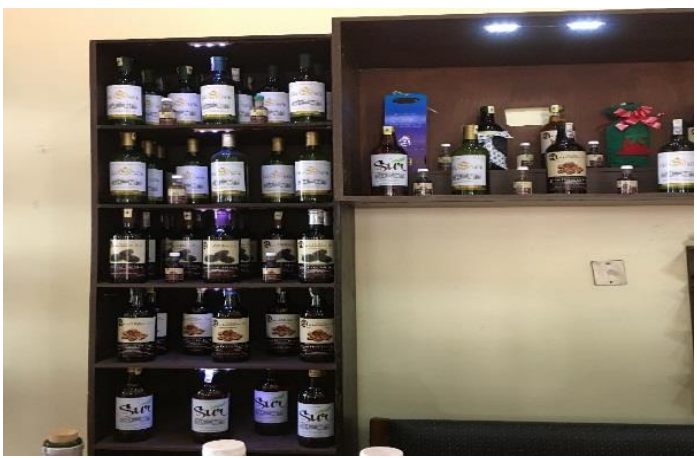

Figura 4. Productos de la empresa Licores y Vinos del Sur de Vilcabamba, Loja

\section{Segunda Fase: Eventos de Capacitación}

Las temáticas programadas en la Segunda Fase (Cuadro 2) se cumplieron en la Universidad Internacional del Ecuador Extensión Loja (UIDE-Loja), en el período del 22 al 28 de marzo de 2018, con el uso de los recursos audiovisuales correspondientes.

Tabla 2. Eventos de capacitación y temas desarrollados

\begin{tabular}{ll}
\hline \multicolumn{1}{c}{ Eventos } & \multicolumn{1}{c}{ Temática } \\
\hline Primero: & 1. Producto, o servicio, presentación \\
& 2. Marca \\
& 3. Logo \\
& 4. Slogan \\
& 5. Medios de distribución y venta. \\
& 6. Incursión en mercado digital. \\
1. Estrategias avanzadas de marketing y comunicación & 2. Estrategia digital y planificación de medios tradicionales y digitales \\
\hline
\end{tabular}

Fuente: Informe de capacitación marzo 2018 


\section{Resultados}

En la primera fase se efectuó un diagnóstico puntual de las empresas y de sus administradores, relacionado con la información de sus productos, de la parte legal, de su estructura y organización.

Tabla 3. Síntesis de las características empresariales de las Mipymes adscritas a ProEcuador

\begin{tabular}{|c|c|c|c|c|c|c|}
\hline No. & Razón social & $\begin{array}{c}\text { Marca } \\
\text { Registrada }\end{array}$ & Logo & Slogan & Mercado & $\begin{array}{c}\text { Marketing } \\
\text { digital }\end{array}$ \\
\hline 1 & \begin{tabular}{l}
\multicolumn{1}{c}{ Alimentos } \\
procesados y \\
marinados del \\
campo
\end{tabular} & No & \begin{tabular}{l}
\multicolumn{1}{c}{ Cuy, } \\
gallina y \\
pato \\
marinado
\end{tabular} & No tiene & \begin{tabular}{l}
\multicolumn{1}{c}{ Local y } \\
nacional bajo \\
pedido
\end{tabular} & $\begin{array}{l}\text { Facebook y } \\
\text { WhatsApp }\end{array}$ \\
\hline 2 & $\begin{array}{l}\text { Sombreros } \\
\text { de paja } \\
\text { toquilla }\end{array}$ & No & Fotografía & No tiene & Local & $\begin{array}{l}\text { Facebook, } \\
\text { WhatsApp, } \\
\text { Twitter }\end{array}$ \\
\hline 3 & APEOSAE & - & - & - & Exportación & $\begin{array}{l}\text { Facebook, } \\
\text { WhatsApp, } \\
\text { Twitter }\end{array}$ \\
\hline 4 & Happy Fruits & En trámite & $\begin{array}{l}\text { En } \\
\text { trámite }\end{array}$ & - & Local & $\begin{array}{l}\text { Facebook, } \\
\text { WhatsApp, } \\
\text { Twitter }\end{array}$ \\
\hline 5 & $\begin{array}{l}\text { Vinos y } \\
\text { Licores del } \\
\text { Sur }\end{array}$ & Sí tiene & $\begin{array}{c}\text { Vinos y } \\
\text { Licores del } \\
\text { Sur } \\
\end{array}$ & - & $\begin{array}{l}\text { Local y } \\
\text { nacional bajo } \\
\text { pedido }\end{array}$ & $\begin{array}{l}\text { Facebook, } \\
\text { WhatsApp, } \\
\text { Twitter }\end{array}$ \\
\hline
\end{tabular}

Fuente: Informe de capacitación marzo 2018

Se explicaron las partes importantes de los productos y servicios: la marca, que en la actualidad se difunde en la web; el logo, para que el negocio tenga su presencia en el mercado; el slogan, que tiene que ser el mensaje correcto, dirigido a la persona correcta y en tiempo correcto; mecanismos que se utilizan para la distribución y venta; y, el manejo de las herramientas del marketing digital. Es algo que podemos aplicar a través de las redes sociales y social media, como canal de comunicación utilizando la creatividad para todas las acciones de estrategias empresariales efectivas, aprovechando el potencial de las redes sociales, para comunicarnos con los clientes, estableciendo relaciones permanentes y de fidelización.

Las empresas deben tener los objetivos claros para definir canales y tácticas de comunicación para ingresar en los mercados, mejorando la rentabilidad y optimizar las inversiones realizadas en los negocios, necesitan utilizar herramientas con contenidos atractivos para ofrecer sus productos directamente a través de la web, posicionando su marca con las nuevas tecnologías como parte importante en los negocios, el cliente es cada vez más exigente y le gusta estar informado en las diversas tecnologías y dispositivos digitales para realizar sus compras, comparar precios y disponibilidad de los productos.

\section{Conclusiones}

Los directivos de las Mipymes necesitan apoyo, seguimiento, asesoramiento y capacitación continua, por ser personas que desconocen las herramientas de marketing digital. 
Para la ejecución de inversiones y la promoción de exportaciones requieren de un curso avanzado de capacitación, para que puedan impulsar la oferta de productos tradicionales y no tradicionales.

Se deben reforzar las capacitaciones, para potenciar y desarrollar competencias en el ámbito de la comercialización y negociación.

Las empresas, con sus respectivos productos, no se encuentran en la capacidad de exportar, necesitan desarrollar su marca, presentación de producto, logo, slogan, empaques y regirse a las leyes de exportación.

Las conclusiones, relacionadas a cada empresa, son:

1. Alimentos Procesados Marinados, tiene una capacidad para exportar de 500 cuyes por mes, pero necesita encontrar un nicho de mercado para este producto; los productos gallina y pato son para determinado segmento de mercado local, su producción es baja y para exportar requiere asesoramiento técnico en cuanto al manejo de aves de corral.

2. Sombreros de Paja Toquilla tiene una producción de 100 sombreros por mes, necesita de herramientas para completar su producción, no dispone de prensadora, y requiere contratar y capacitar personal para aumentar su producción.

3. APEOSAE, que es la Asociación de Productores Ecológicos y Orgánicos del Sur de la Amazonía Ecuatoriana, lleva varios años en el mercado internacional y el principal destino de exportación de sus productos es Francia.

4. Happy Fruits tiene como producto principal un snack de 40 gramos de fruta deshidratada, de mango y pitahaya; necesita cambiar la presentación del producto y buscar nichos nacionales.

5. Vinos y Licores del Sur es una empresa familiar, su producción es limitada, necesita transformar la capacidad de la planta procesadora de manual a industrial.

\section{Bibliografía}

Armijos, N. (2018). Proyecto diseño e implementación de estrategias de marketing y comunicación para Mipymes: informe. Loja, Ecuador: Universidad Internacional del Ecuador - Loja.

Cohen, W. (1992). Plan de marketing. Bilbao, España: ESIC Editorial

Esteban Talaya, A. y Lorenzo Romero, C. (Coord.). (2013). Dirección comercial. Madrid, España: ESIC Editorial. 
Fernández, C. C. (2000). El plan de marketing: cómo elaborarlo con ayuda informática. $3^{\mathrm{a}}$ ed. Madrid, España: ESIC Editorial.

González, B. A., Román, P. e Idoeta, A. M. C. (2014). Introducción al marketing y la comunicación en la empresa. Madrid, España: ESIC Editorial.

Kotler, P., Keller, K. L. (2012), Dirección de marketing. 14ª ed. México: Pearson Educación.

Marketing, P. (1999). El plan de marketing personal. Recuperado de

https://ebookcentral.proquest.com

Sainz de Vicuña Ancín, J. M. (2017). El plan de marketing digital en la práctica. $2^{\mathrm{a}}$ ed. Madrid, España: Esic Editorial.

Sainz de Vicuña Ancín, J. M. (2016). El plan de marketing en la Pyme. $3^{\mathrm{a}}$ ed. Madrid, España: ESIC Editorial.

Sainz de Vicuña Ancín, J. M. (2015). El plan de marketing en la práctica. 10ª ed. Madrid, España: ESIC Editorial.

Santesmases, M. (2000). Marketing, concepto y estrategias. Madrid, España: Pirámide.

Soria, I. M. D. M. (2017). Plan de marketing empresarial: uf2392. Recuperado de https://ebookcentral.proquest.com 